\title{
INTERVIEW
}

For reprint orders, please contact: reprints@futuremedicine.com

\section{Fourty years of dedication to chronic myeloid leukemia}

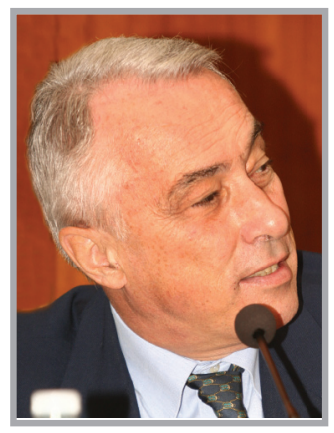

Michele Baccarani* speaks to Roshaine Gunawardana, Managing Commissioning Editor: Michele Baccarani MD, obtained his degree from the University of Bologna in 1966 and received his postgraduate degree in Hematology at the University of Modena in 1969. He has held various positions during his career, including Research fellow at Rigshospitalet, University of Copenhagen, Professor of Hematology at the University of Udine and Head of the Institute of Hematology "L. and A. Seràgnoli", S.Orsola-Malpighi University Hospital, Bologna. He was also the Vice-Dean of the Faculty of Medicine and Surgery at the University of Bologna from 2001 to 2007. Professor Baccarani has held posts as President of the Italian Society of Experimental Hematology and President of the Italian Society of Hematology, and is currently the Head of the Department of Hematology and Oncology " $\mathrm{L}$ and A Seràgnoli" S Orsola-Malpighi University Hospital, Bologna. He has been the Chairman of the GIMEMA CML Working Party from 1989 and is a member of the Working Package 4 Chronic Myeloid Leukemia and of the European LeukemiaNet. $\mathrm{He}$ is the author or coauthor of more than 500 papers published in peer-reviewed journals. Professor Baccarani's clinical interests include chronic myeloid leukemia, acute leukemia, chemotherapy and stem cell transplantation, while his research interests comprise cell kinetics, stem cells, multidrug resistance and the molecular pathogenesis of leukemia.

\section{What led to your specific interest in} the field of hematologic oncology?

When I was still a student, during an internship in internal medicine, I had the chance to observe and follow a case of acute leukemia in a radiologist. At that time it was not easy to look directly at cancer cells. I could observe, day by day, the leukemic cells under the microscope; I was fascinated and I understood that leukemia was a key to the understanding of cancer.

\section{What led to your specific interest in} chronic myeloid leukemia?

I have always had a strong interest in all blood malignancies and in stem cell transplantation. Chronic myeloid leukemia
(CML) became my favorite because it was a model mapping out the road to the development of acute leukemia. CML itself is a 'benign' disease, like a preleukemia, but it led to the death of all the patients, without any exception. As a young doctor, I could not tolerate the idea that a patient who was relatively well was destined to die in almost all cases.

\section{Q Could you briefly describe the} research you are conducting at present \& the clinical implications of such research? For many years I tried to study the cell kinetics of $\mathrm{Ph}^{+}$cells, to understand and identify the leukemic stem cells and their properties. These studies were not very successful until the development of the
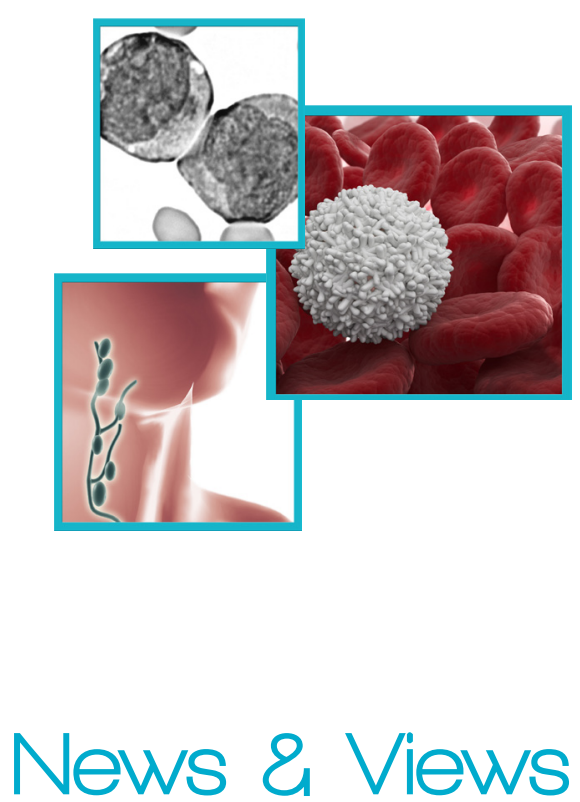

News

Journal Watch

Interview

*Department of Hematology \& Oncology "L \& A Seràgnoli", S Orsola-Malpighi University Hospital, University of Bologna, Via Massarenti 9, 40138 Bologna, Italy; Tel.: +39 051 390413; Fax: +39 051 398973; michele.baccarani@unibo.it

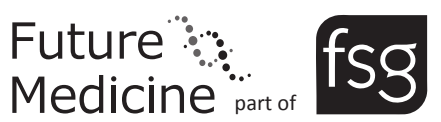


appropriate techniques, particularly of in vitro cultures. To make a long story short, these studies contributed to establish and confirm that CML could not be successfully treated with any type of conventional cytotoxic chemotherapy. I did not give any personal contribution to the identification and the development of IFN- $\alpha$ and of the tyrosine kinase inhibitors (TKIs), but I contributed to the best of my abilities to their introduction and clinical application, in Phase I, II and III studies, and to the understanding of their proper use, developing internationally recognized guidelines and recommendations. For this purpose, I had the honor to chair an international panel of experts appointed by European LeukemiaNet.

You are the coordinator of the Coop Group Italian Association for the study of CML. What specifically is this group involved in?

The Italian Cooperative Study Group on CML, now the GIMEMA CML Working Party (GIMEMA is the Italian Group for the Hematologic Malignancies in Adults), was established in 1973. I began to serve as a secretary, then in 1995 I became the chair of the group. The group includes almost all the University and General Hospitals in Italy (more than 80) and has performed studies of treatment, from experimental chemotherapy and splenectomy, to interferon and TKIs. IFN- $\alpha$ was registered in the USA by the US FDA for the treatment of CML based on a study that was promoted, sponsored and performed by the Italian Group. The Group is currently taking care of several trials of TKIs, and has developed a network of laboratories (LabNet) that ensures a standardized measurement of molecular response, based on the International Scale.

\section{How have you seen the field of} hematologic malignancies evolve over the years?

Major progress was initiated 40 years ago, in the 1970s, when malignant lymphomas, mainly Hodgkin's disease, and acute leukemias, mainly acute lymphoblastic leukemias of children, became curable. These successes were based on conventional cytotoxic drugs. Next, TKIs, all-trans retinoic acid and monoclonal antibodies have paved the way to success in other diseases, a way that is still in its initial stages.

\section{As an experienced teacher \&} professor, what would you consider to be the most important areas in which education in the field of hematologic malignancies can be improved?

The 'new' hematologist must have in their pocket a modern textbook of 'molecular hematology' and an old textbook of internal medicine, to be able to recognize the molecular basis of the disease, to optimize the treatment and to take care of the patient and not only the disease.

What do you think will be the most important topics in the field of hematologic malignancies over the next few years?

I share the opinion of many of my colleagues that the future will see an explosion of the so-called targeted therapy. However, I believe that the times are mature enough to make a breakthrough in allogeneic stem cell transplantation, which means resolving the problems of the transplantation and the adaptation of the immune system from one person to another.

\section{Financial \& competing interests}

\section{disclosure}

$M$ Baccarani has received honoraria for speaker bureau and advisory board contributions from Novartis Pharma, Bristol-Myers Squibb, Pfizer and Ariad. M Baccarani has no other relevant affiliations or financial involvement with any organization or entity with a financial interest in or financial conflict with the subject matter or materials discussed in the manuscript apart from those disclosed.

No writing assistance was utilized in the production of this manuscript. 